\title{
A NEW CONCEPT FOR IMPLANT FIXATION: BONE-TO-BONE BIOLOGIC FIXATION
}

\author{
D.-Y. Kim ${ }^{1}$, J.-R. Kim², K.Y. Jang ${ }^{3}$ and K.-B. Lee ${ }^{2 *}$
}

\author{
${ }^{1}$ Department of Orthopaedic Surgery, Yeolin hospital, Iksan, Korea \\ ${ }^{2}$ Department of Orthopaedic Surgery, Research Institute of Clinical Medicine of Chonbuk National University- \\ Biomedical Research Institute of Chonbuk National University Hospital, \\ Chonbuk National University Medical School, Jeonju, Korea \\ ${ }^{3}$ Department of Pathology, Chonbuk National University Medical School, Jeonju, Korea
}

\begin{abstract}
Many attempts have been made to reduce complications of bone implant, such as pedicle screw loosening. To address this problem, the authors suggest a new concept of boneto-bone biologic fixation using recombinant human bone morphogenetic protein-2 (rhBMP-2)-loaded cannulated pedicle screws. Recombinant human bone morphogenetic protein-2 is an osteoinductive cytokine. Four types of titanium pedicle screws were tested (uncannulated, cannulated with no loading, beta-tricalcium phosphate (TCP)-loaded, and TCP/BMP2 loaded) using 16 miniature pigs. Radiological evaluation was conducted to assess the fusion and loosening of pedicle screws. Twelve weeks after implantation, peak torsional extraction torque was measured, and the pedicle screw and bone interface was evaluated by micro-computed tomography $(\mu \mathrm{CT})$ and histologic examination. The mean value of the radiological score was significantly greater in the TCP/BMP2 loaded group at 12 weeks post-operation compared to those in the other groups. CT images showed distinct bone formation surrounding TCP/BMP2 loaded cannulated pedicle screws compared to the other groups. Mean extraction torsional peak torque at 12 weeks postoperative was more than 10-fold higher in the TCP/BMP2 loaded pedicle screw group than in the other groups. Bone surface and bone volume, as quantitated through $\mu \mathrm{CT}$, were higher in the TCP/BMP2 loaded group. Histologic examination revealed bone-to-bone fixation at the interface of pedicle screws and pre-existing bone. Bone-to-bone biologic fixation through the holes of TCP/BMP2 loaded pedicle screws significantly increased fixation strength and represents a novel method that can be applied to osteoporotic or tumour spine surgeries.
\end{abstract}

Key Words: Pedicle Screw; rhBMP; bone-to-bone fixation.

*Address for correspondence:

Dr. Kwang-Bok Lee

Department of Orthopaedic Surgery, Research Institute of Clinical Medicine of Chonbuk National University-

Biomedical Research Institute of Chonbuk National

University Hospital, Chonbuk National University

Medical School

634-18 Keumam-dong, Jeonju-shi, Chonbuk-do,

South Korea

Telephone number: +82-63-250-1760

Fax number: +82 -63-271-6538

E-mail: osdr2815@naver.com

\section{Introduction}

Pedicle screw fixation is currently the gold standard for the treatment of spinal deformities, degenerative conditions of the lumbar spine, traumatic fractures, and reconstruction after spinal tumour resection (Greenfield et al., 1992; McLain et al., 2001; Upasani et al., 2009; Zeiller et al., 2005). Several clinical studies have demonstrated that instrumentation with pedicle screws increases the bone fusion rate compared to those of non-instrumented techniques (Sandén et al., 2001; Yuan et al., 1994; Zdeblick et al., 1993). Pedicle screws allow for control of all three spinal columns from a posterior-only approach and have been reported to have numerous advantages over traditional hook and wire instrumentation (Butler et al., 1994; Ferguson et al., 1988; Goel et al., 1988; Hitchon et al., 2003).

Rigid fixation between a pedicle screw and bone depends on the quality of the bone and the type of screw utilised. Pedicle screw loosening, especially in cases of osteoporotic vertebrae and skeletal tumours, is a major concern among spine surgeons (Frankel et al., 2007; Halvorson et al., 1994; Weinstein et al., 1988). Many attempts have been made to address this complication.

In order to improve sustained fusion of pedicle screws to the bone, different surgical instrumentation methods have been attempted, including alterations in thread design and screw shape, as well as surface modifications of the screws to enhance bone apposition (DeCoster et al., 1990; Krag et al., 1986; Kwok et al., 1996; Lapresle et al., 1995; Sell et al., 1988). However, these pedicle screw designs have not provided the desired results in reducing the incidence of pedicle screw loosening.

We inserted the osteoinductive cytokine recombinant human bone morphogenetic protein-2 (rhBMP-2) into cannulated pedicle screws, expecting to produce osseointegration through rhBMP-2-induced bone formation both around the screw and continuous with bone formation through the holes in the screw (so called "bone-to-bone fixation"). The aim of this study was to evaluate this approach using radiological, mechanical, and histological methods. No previous study has reported the use of rhBMP-2-loaded pedicle screws to enhance the bond interface between pedicle screws and bone. The authors suggest a new concept of using rhBMP-2-loaded pedicle screws to provide high fixation strength. 
Fig. 1. Photographs of the pedicle screws. A: Photograph of a cannulated pedicle screw. Cannulated pedicle screws were made of Ti-6Al-4V ELI alloy with an outer diameter of $4.5 \mathrm{~mm}$ and a length of $25 \mathrm{~mm}$. Screws were fabricated with one cannulation along the long axis of the screw and five holes in the thread. B: Immediate post-operative plain radiographs showing the pedicle screws and rods at L4-5 vertebrae in miniature pigs.

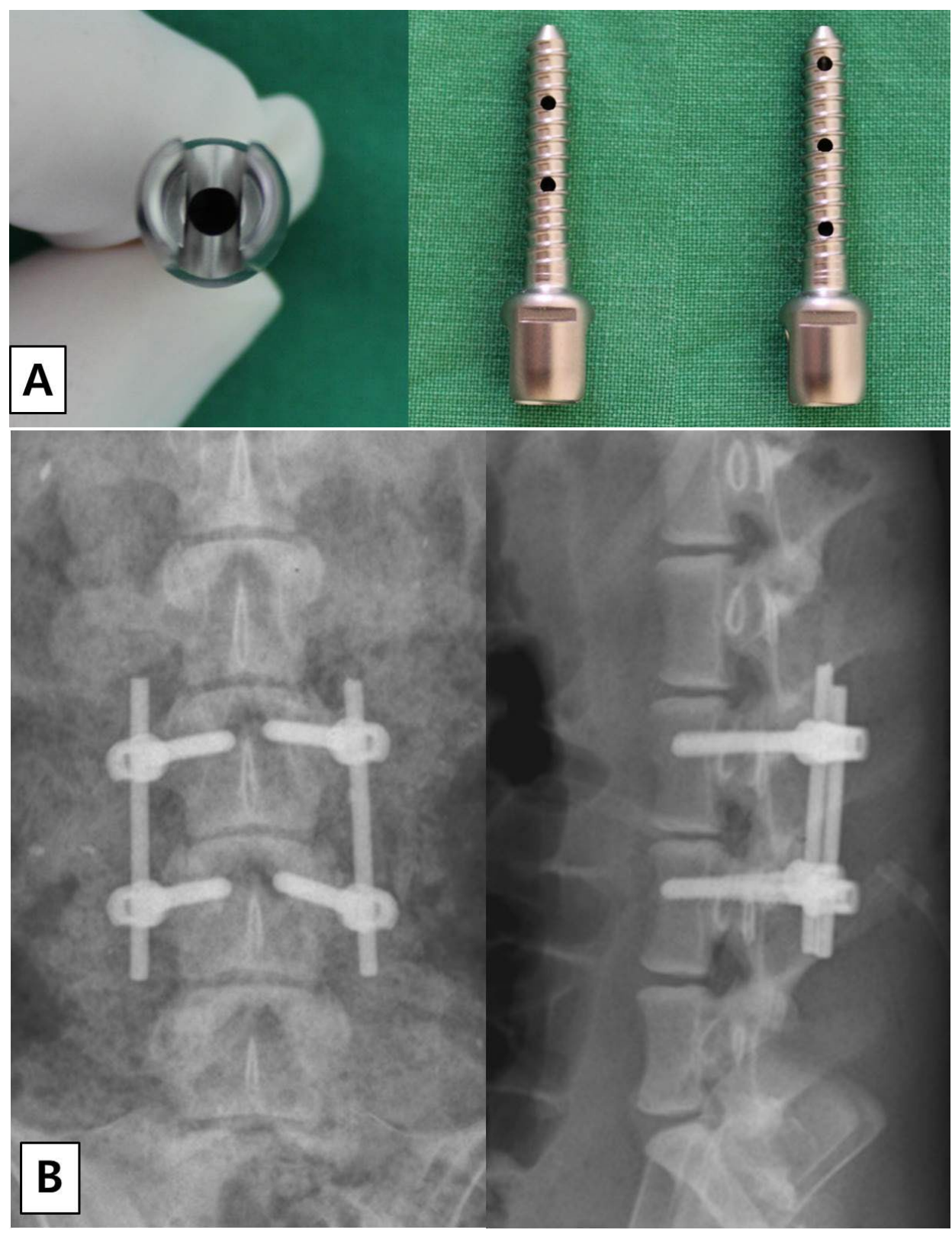

\section{Materials and Methods}

\section{Study design and implant materials}

Sixteen mature female miniature-pigs, approximately one year old and weighing approximately $50 \mathrm{~kg}$, were used in this study. This study was approved by the Institutional Animal Care and use Committee of Chonbuk National University. Animal subjects were divided into four groups with respect to the type of pedicle screw used. Each group consisted of four miniature pigs. The groups were as follows:

1. Uncannulated: an ordinary screw that had no cannulation

2. Cannulated but not loaded (cannulated): screws the same size and material as uncannulated screws but with cannulation in the centre of the long axis and five holes in the tread area (Fig. 1-A)

3. Cannulated and loaded with beta-tricalcium phosphate (TCP): cannulated screw loaded with beta-tricalcium phosphate
4. Cannulated and loaded with TCP and rhBMP-2 (TCP/ BMP2): cannulated screw loaded with TCP and rhBMP-2.

A posterior fixation system (CGBio Co., Ltd. Seongnam, Gyonggido, Korea) was used for instrumentation. The monoaxial pedicle screws (uncannulated) designed for this study were made of Ti-6Al-4V ELI alloy with an outer diameter of $4.5 \mathrm{~mm}$ and a length of $25 \mathrm{~mm}$. The other monoaxial pedicle screws (cannulated) were the same size and material as the uncannulated screws but had a cannulation in the centre of the long axis and five holes in the tread area (Fig. 1-A). Rods made of Ti-6Al-4V ELI alloy were used as monosegmental constructs connecting the upper and lower pedicle screws.

\section{Surgery and implantation procedure}

At total of 64 pedicle screws were implanted into 16 miniature pigs with two-level instrumentation, using four pedicle screws per animal. Subjects were kept $N P O$ 
(nothing by mouth) for $12 \mathrm{~h}$ prior to surgery. Subcutaneous prophylactic antibiotics were administered prior to surgery. The miniature pigs were immobilised in the supine position, and the surgical area was shaved and disinfected with alcohol and povidone-iodine. Anaesthesia was induced with sodium pentothal, and intubated subjects were maintained with nitrous oxide and $2 \%$ isoflurane.

A longitudinal midline incision was made to expose the posterior elements and transverse processes. After ensuring cortical integrity of the pedicle and vertebral body with a pedicle probe tapper, pedicle screws were inserted bilaterally to the fourth and fifth lumbar vertebrae. A C-arm image intensifier was used to confirm the pedicle screw positions. Screws in the appropriate position were then loaded with test materials. For the beta-TCP-loaded group, each screw was injected with $0.8 \mathrm{~mL}$ of a solution of $0.5 \mathrm{~mL}$ beta-TCP and poloxamer and $0.3 \mathrm{~mL}$ normal saline. For the TCP and rhBMP-2-loaded group, each screw was injected with $0.8 \mathrm{~mL}$ of a solution of $0.5 \mathrm{~mL}$ betaTCP and poloxamer and $0.3 \mathrm{~mL}(0.75 \mathrm{mg} / \mathrm{mL}) \mathrm{rhBMP}-2$ (concentration: $2.5 \mathrm{mg} / \mathrm{mL}$, Daewoong Pharmaceutical Co. Ltd., Seoul, Korea). Normal saline was used for irrigating hematomas, devitalised soft tissue, and bone fragments. A drain was placed, and subcutaneous tissue and skin were closed with absorbable sutures and non-absorbable sutures, respectively. Subcutaneous analgesia and antibiotics were administered for seven days postoperatively.

\section{Radiological assessment}

Immediate postoperative radiological evaluation with X-ray (Fig. 1B) and CT (Siemens Emotion 16, Siemens Manufacturing Co., Inc., Forchheim, Germany) was performed to ensure proper placement of pedicle screws and rods. Follow-up radiological evaluations were performed with X-ray and CT at 12 weeks after surgery. For evaluation of bone formation around the inner and outer surfaces of the pedicle screws and the degree of pedicle screw loosening, all radiological images were reviewed by an independent orthopaedic surgeon blinded to treatment assignments. The incidence of loosening was compared between groups at post-operative 12 weeks. Loosening was defined as the appearance of radiolucent zones surrounding the pedicle screw or evidence of pullout or a change in screw placement status compared to immediate postoperative CT scans (Sandén et al., 2004; Abul-Kasim et al., 2014).

\section{Postharvest procedure and mechanical testing}

The miniature pigs were sacrificed at 12 weeks after surgery, and the lumbar spines from L4 to L5 were resected en bloc. The spinal specimens were extracted with an oscillating saw at the desired levels of the intervertebral body. Soft tissues from the fresh specimens were removed with extra caution to not transmit any force to the pedicle screws. Forty-eight screws from 12 miniature pigs, representing 3 of the 4 miniature pigs from each group, were prepared for mechanical testing. Mechanical testing of torsional screw extraction torque was performed using an AIKOH testing gauge (Model 9800 series, AIKOH Engineering Co., Tokyo, Japan) directly on the fresh spines.
Securing the vertebral bone-implant block into the holder was followed by screwing a custom-fabricated driver tip into the pedicle screw head. The torsional screw extraction was carried out at a uniform rate to a maximum excursion of $30^{\circ}$. Peak torque was recorded in $\mathrm{N} \mathrm{m} / 100$.

\section{Quantitative evaluation using $\boldsymbol{\mu - C T}$}

At 12 weeks, one subject from each group was randomly selected for high-resolution $\mu-\mathrm{CT}$ imaging and histologic evaluation of the interface between the pedicle screw and bone. CT analysis was performed using a SkyScan instrument (SkyScan1173; SKYSCAN, Kartuizersweg 3B 2550 Kontich, Belgium). Measurements of bone volume fraction, bone surface density, and specific bone surface were obtained from the surrounding of pedicle screws. Bone volume fraction (bone volume/total volume, \%) was defined as the ratio of segmented bone volume to the total bone volume of the region of interest. Bone surface density (bone surface/total volume, $\mathrm{mm}^{2} / \mathrm{mm}^{3}$ ) was defined as the ratio of segmented bone surface to the total bone volume of the region of interest. Specific bone surface (bone surface/ bone volume, $\mathrm{mm}^{2} / \mathrm{mm}^{3}$ ) was defined as the ratio of segmented bone surface to the segmented bone volume.

\section{Histological evaluation}

Following $\mu$-CT imaging, specimens of the L4 and L5 vertebrae with implantation were excised and fixed in $10 \%$ buffered formaldehyde. They were then dehydrated in a graded series of $70 \%, 80 \%, 90 \%$ and $100 \%$ ethanol, increasing the concentration daily, and embedded in methylmethacrylate. After polymerisation, the specimens were cut along the long axis of the pedicle screws using the EXAKT diamond cutting system (EXAKT $300 \mathrm{CP}$, Germany). The sections were stained with Goldner Trichrome and Haematoxylin-Eosin and were examined under light microscopy (BX51, OLYMPUS, Japan).

\section{Statistical analysis}

Statistical analysis was carried out with the Statistical Package for Social Sciences software (SPSS Inc., Seoul, Korea). The data of radiological evaluation (the incidence of loosening) was analysed using Chi-square test. Data pertaining to mechanical testing (extraction torsional peak torque), and quantitative data from $\mu$-CT were analysed using one-way ANOVA test with post-hoc test (Scheffe test) for comparison between TCP/BMP2 loaded cannulated screw and other control groups (uncannulated, cannulated, and TCP loaded screw). $p$ values less than 0.05 were considered to be statistically significant.

\section{Results}

A total of 16 miniature pigs underwent implantation surgery, and no major complications were noted during or after surgery. One miniature pig from the uncannulated group developed a wound infection at the superficial surgical site but completed the study in satisfactory condition. No metal failure such as breakage or disconnection of pedicle screws or rods was observed. 


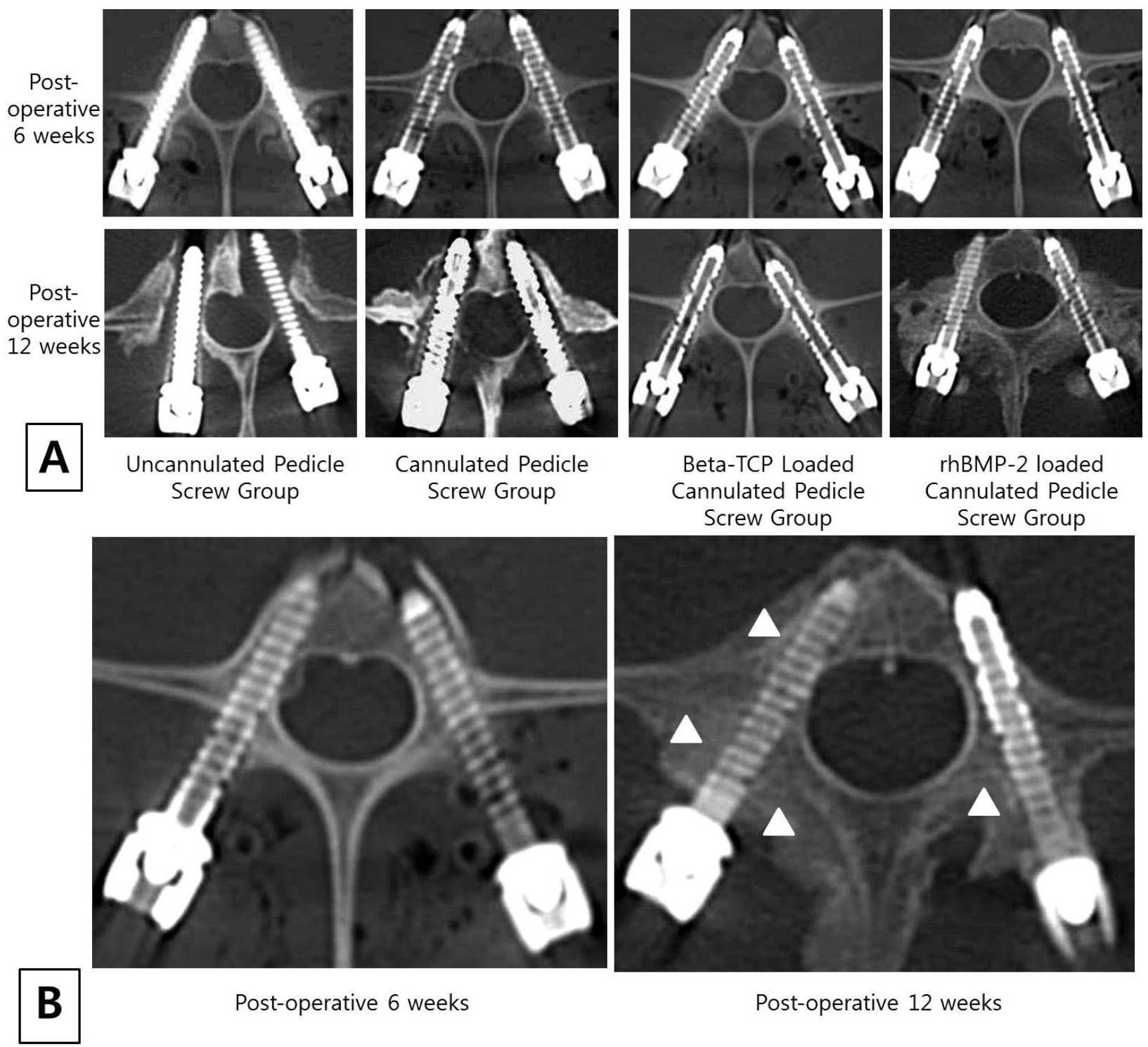

Fig. 2. Radiographic analyses. A: CT axial image of L-spine CT illustrating new bone formation in and around the screw in the TCP/BMP2 loaded group. White arrowheads denote rhBMP-2 induced new bone formation around pedicle screws, forming a bulky mass. Note the loosening of screws in the uncannulated, cannulated but unloaded, and TCP loaded groups at six and 12 weeks but the absence of such loosening in screws from the TCP/BMP2 loaded group. B: Illustration of a case of screw loosening in the TCP/BMP2 loaded group at six weeks, which resolved and produced rigid fixation between the screw and the host bone by 12 weeks.

Table 1. The incidence of loosening between groups.

\begin{tabular}{|l|c|c|c|}
\hline & $\begin{array}{c}\text { No } \\
\text { loosening }\end{array}$ & Loosening & $\begin{array}{c}\text { Chi-square } \\
\text { test }\end{array}$ \\
\cline { 1 - 3 } Uncannulated screw $(n=12)$ & 2 & 10 & \multirow{2}{*}{$p=0.002$} \\
\cline { 1 - 2 } Cannulated screw $(n=12)$ & 6 & 6 & \\
\cline { 1 - 2 } TCP-loaded cannulated screw $(n=12)$ & 8 & 4 & \\
\cline { 1 - 2 } TCP/BMP2 loaded cannulated screw $(n=12)$ & 11 & 1 & \\
\cline { 1 - 2 }
\end{tabular}

*Loosening indicates partial, total loosening or screw pull-out.

\section{Radiological assessment}

The incidence of loosening between groups is shown in Table 1. There was a statistically significant difference between TCP/BMP2 loaded screw group and other groups at 12 weeks after surgery, TCP/BMP2 loaded screw had a lower loosening rate than other groups (Chi-square test, $\chi^{2}=14.5, p=0.002$, Table 1).
Images from the L-spine $\mathrm{CT}$ examination are presented in Fig. 2B and 2C. These images show severe screw loosening and no evidence of bone formation in the uncannulated, cannulated, or TCP-loaded groups. However, they also show abundant bone formation around screws from the TCP/BMP2 loaded group. In addition, there was a single case of partial screw loosening in 


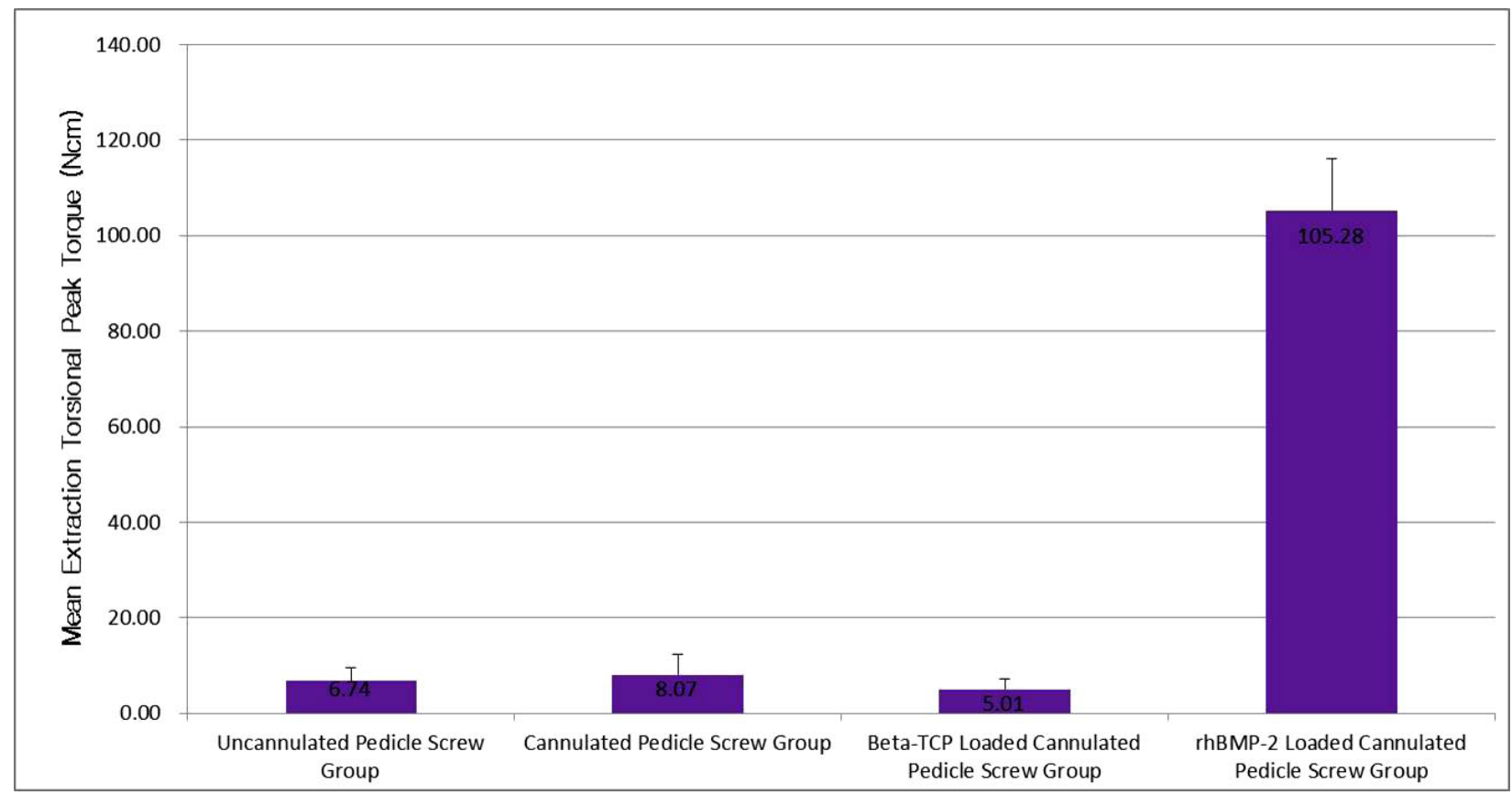

Fig. 3. Biomechanical testing. Mean (and s.e.) extraction torsional peak torque for each group at 12 weeks. The value for the TCP/BMP2 loaded group was significantly greater than those of the other groups (one-way ANOVA, $p<0.001$. post-hoc test with Scheffe, uncannulated $v s$. cannulated, $p=0.966$; uncannulated $v s$. TCP, $p=0.929$, uncannulated $v s$. TCP/BMP2, $p<0.001$; cannulated $v s$. TCP, $p=0.706$; cannulated $v s$. TCP/BMP2, $p<0.001$, TCP vs. TCP/BMP2, $p<0.001)$.

the TCP/BMP2 loaded group six weeks after surgery. However, by 12 weeks, a firm bond between this screw and the surrounding bone had formed (Fig. 2C). More frequent and more severe screw loosening was observed in all of the three control groups.

\section{Mechanical testing}

The results of the biomechanical testing performed after 12 weeks are presented in Fig. 3. Mean (and s.e.) values were as follows: uncannulated $(0.0674 \pm 0.028 \mathrm{~N} \mathrm{~m})$, cannulated $(0.0807 \pm 0.0433 \mathrm{~N} \mathrm{~m})$, TCP loaded $(0.0807 \pm 0.0433 \mathrm{~N} \mathrm{~m})$, and TCP/BMP2 loaded (1.0528 $\pm 0.1087 \mathrm{~N} \mathrm{~m})$. Mean extraction torsional peak torque was significantly higher in the TCP/BMP2 group than other groups (one-way ANOVA, $p<0.001$. post-hoc test with Scheffe, uncannulated $v$ s. cannulated, $p=0.966$; uncannulated $v s$. TCP, $p=0.929$, uncannulated vs. TCP/BMP2, $p<0.001$; cannulated $v s$. TCP, $p=0.706$; cannulated vs. TCP/BMP2, $p<0.001$, TCP vs. TCP/BMP2, $p<0.001$, Fig. 3).

\section{Quantitative $\mu$-CT evaluation}

Quantitative data from the $\mu$-CT evaluation after 12 weeks are shown in Fig. 4.

The value for the TCP/BMP2 group in bone volume fraction was significantly greater than those of the other groups (one-way ANOVA, $p<0.001$. post-hoc test with Scheffe, uncannulated vs. cannulated, $p=0.722$; uncannulated $v s$. TCP, $p=0.168$, uncannulated $v s$. TCP/BMP2, $p<0.001$; cannulated vs. TCP, $p=0.657$; cannulated $v s$. TCP/BMP2, $p<0.001, \mathrm{TCP} v s$. TCP/BMP2, $p<0.001$, Fig. 4-A).
The value for the TCP/BMP2 group in bone surface density was significantly greater than those of the other groups (one-way ANOVA, $p<0.001$. post-hoc test with Scheffe, uncannulated vs. cannulated, $p=1.000$; uncannulated $v s$. TCP, $p=0.961$; uncannulated $v s$. TCP/BMP2, $p<0.001$; cannulated $v s$. TCP, $p=0.963$; cannulated $v s$. TCP/BMP2, $p<0.001, \mathrm{TCP} v s$. TCP/BMP2, $p<0.001$, Fig. 4-B).

The value for the TCP/BMP2 group in specific bone surface was significantly greater than those of the other groups (one-way ANOVA, $p<0.001$. post-hoc test with Scheffe, uncannulated vs. cannulated, $p=0.704$; uncannulated $v s$. TCP, $p=0.078$; uncannulated $v s$. TCP/BMP2, $p<0.001$; cannulated $v s$. TCP, $p=0.415$; cannulated $v s$. TCP/BMP2, $p<0.001, \mathrm{TCP} v s$. TCP/BMP2, $p<0.001$, Fig. 4-C). Homogenous subset was shown between three control groups.

\section{Histological examination}

Histologic examination after 12 weeks of implantation demonstrated distinct difference among the groups (Fig. 5 ). The spaces between the pedicle screw and host bone were filled only with loose fibrous tissue without notable new bone formation in the uncannulated, cannulated but unloaded, and TCP-loaded groups. The intra-luminal spaces of the cannulated screws in the cannulated but unloaded and the TCP-loaded groups were also filled with loose fibrous tissue without new bone formation. In contrast, dramatic new bone formation was identified in the TCP/BMP2 loaded group. Newly formed mature (lamellar) bone filled the intra-luminal space, and outgrowths to the 

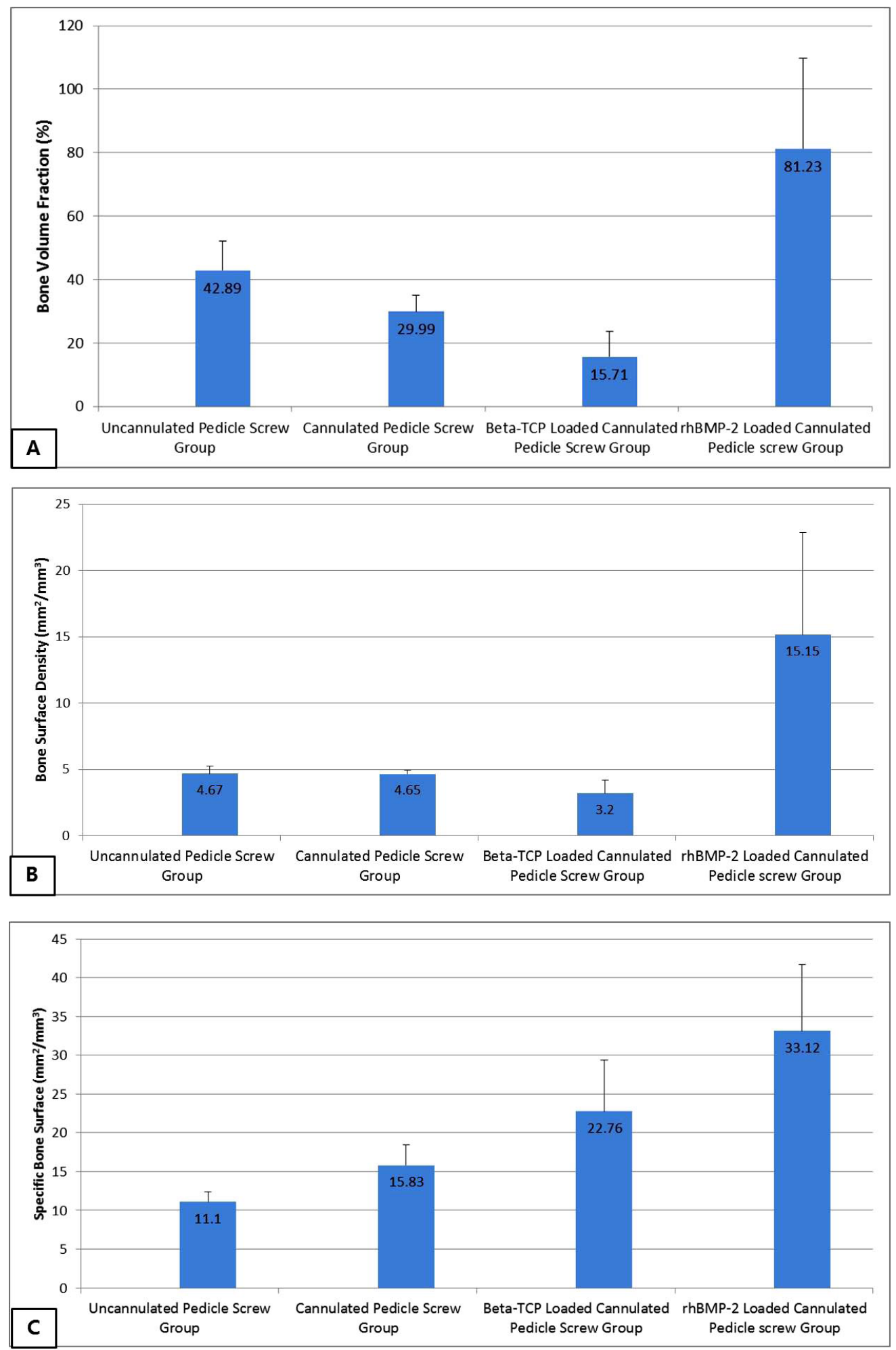

Fig. 4. Quantitative $\mu \mathrm{CT}$ analysis. Mean values of the three parameters for bone formation as quantitated by $\mu \mathrm{CT}$ analysis. Parameters include bone volume fraction, bone surface density, and specific bone surface (A, B and $\mathbf{C}$, respectively). A: The value for the TCP/BMP2 group in bone volume fraction was significantly greater than those of the other groups (one-way ANOVA, $p<0.001$. post-hoc test with Scheffe, uncannulated vs. cannulated, $p=0.722$; uncannulated $v s$. TCP, $p=0.168$, uncannulated $v s$. TCP/BMP2, $p<0.001$; cannulated $v s$. TCP, $p=0.657$; cannulated $v s$. TCP/BMP2, $p<0.001$, TCP $v s$. TCP/BMP2, $p<0.001)$. B: The value for the TCP/BMP2 group in bone surface density was significantly greater than those of the other groups (one-way ANOVA, $p<0.001$. post-hoc test with Scheffe, uncannulated vs. cannulated, $p=1.000$; uncannulated $v s$. TCP, $p=0.961$; uncannulated $v s$. TCP/BMP2, $p<0.001$; cannulated $v s$. TCP, $p=0.963$; cannulated $v s . \mathrm{TCP} / \mathrm{BMP} 2, p<0.001$, TCP $v s . \mathrm{TCP} / \mathrm{BMP} 2, p<0.001)$. C: The value for the TCP/BMP2 group in specific bone surface was significantly greater than those of the other groups (one-way ANOVA, $p<0.001$. post-hoc test with Scheffe, uncannulated $v s$. cannulated, $p=0.704$; uncannulated $v s$. TCP, $p=0.078$; uncannulated $v s$. TCP/BMP2, $p<0.001$; cannulated $v s$. TCP, $p=0.415$; cannulated $v s$. TCP/BMP2, $\mathrm{p}<0.001$, TCP $v s$. TCP/BMP2, $p<0.001$ ). Homogenous subset was shown between three control groups. 


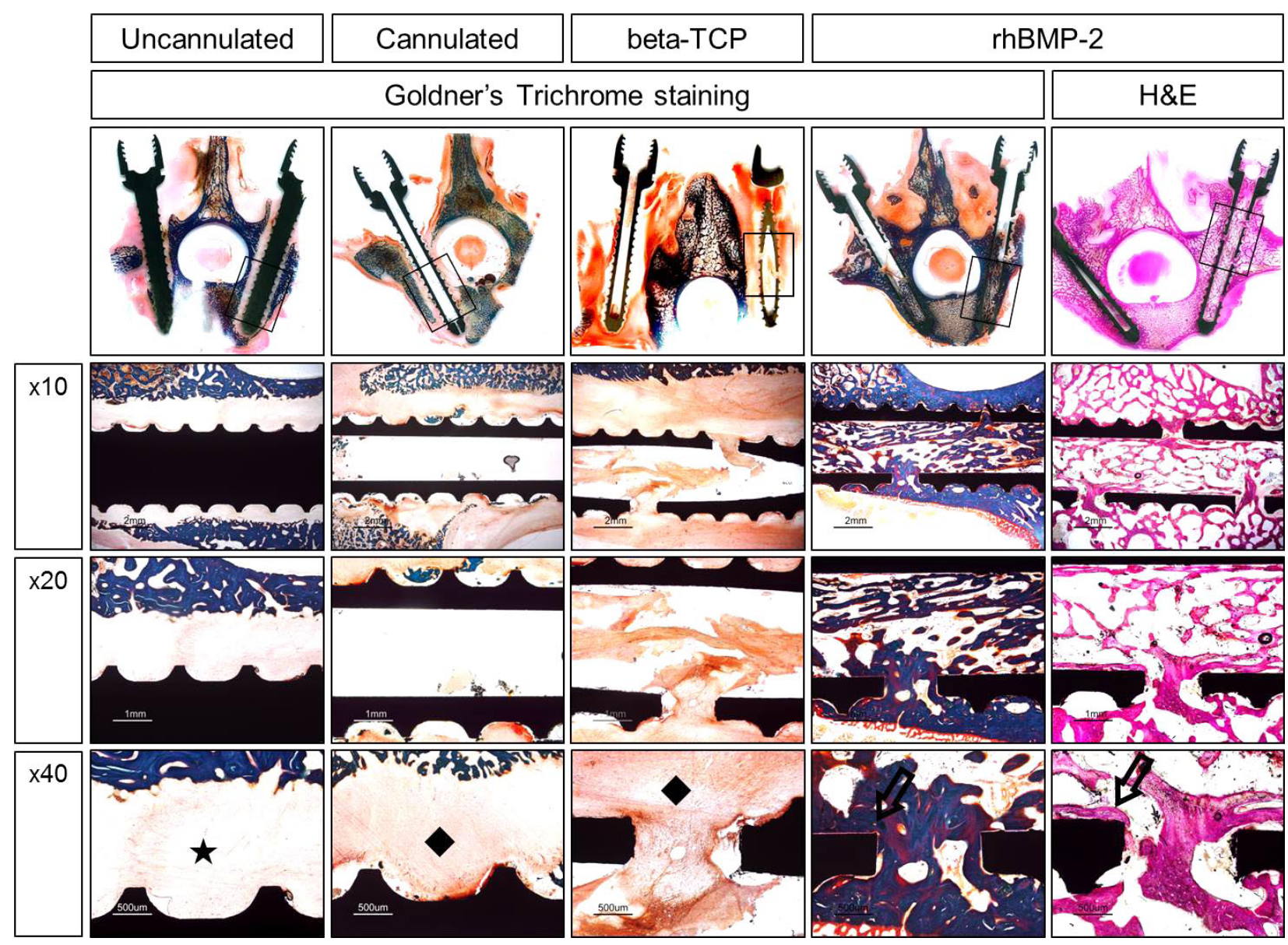

Fig. 5. Histological examination. Histological examination of screws from each group. Note that, in the uncannulated group, the pedicle screw is surrounded by loose connective tissue (asterisk). In the cannulated but not loaded group and the TCP group, the intra-luminal spaces and the spaces around the screws are filled with loose connective tissue without apparent new bone (diamonds). In contrast, in the TCP/BMP2 loaded group, the lumen and the area around the screw are filled with mature (lamellar) bone. These two areas of bone are connected by a solid osseous bridge (open arrow). Original magnification $40 \times$.

outer surface of the cannulated screw through the holes in the screw formed a stout bone bridge between the intraluminal and outer bone surface. The newly formed bone tightly attached to the inner and outer surfaces of the screw and the new bone, filled the gap between the screw and the bone, and created a strong bond at the interface of the pedicle screw and host bone.

\section{Discussion}

Spinal implant surface coatings, such as titanium and hydroxyapatite plasma-spray coatings, have long been studied in effort to provide a more stable interface between the implant and the pre-existing bone, especially osteoporotic bone. These materials are used because they are perceived to increase the fusion success rate. While these techniques are commonly employed, it is evident that they do not provide a complete and satisfactory solution to the clinical problem, especially in situations involving osteoporosis or skeletal tumours. Furthermore, these approaches have various shortcomings. For example, bone ingrowth into titanium plasma-spray-coated implants remains controversial. There are many studies reporting positive results associated with the use of hydroxyapatite plasma-spray-coated implants, but loosening caused by inflammatory reactions and a lack of bonding between the interface of the pedicle screw and the hydroxyapatite plasma-spray-coating remain to be overcome. Primary fixation is one of the most important factors in establishing adequate osseo-integration between bone and an implant fixture (Bouxsein et al., 2010; Ong et al., 2004; Johansson et al., 1987). Unfortunately, there is currently no material that can enhance bone formation in the setting of spinal instrumentation. Therefore, new materials or concepts are required for enhancing the fixation strength between screw and bone.

Data presented in this report demonstrate that the use of TCP/BMP2 loaded cannulated pedicle screws resulted in a significant improvement in the radiological index of screw loosening. All groups showed an increase in index over time, but the difference in the TCP/BMP2 loaded group was minimal. We attribute this to increased bone formation. On the other hand, increased loosening was observed in all 3 control groups, and we associate this with the absence of new bone formation. These findings were 
emphatically reinforced by the fact that biomechanical testing found a greater than 10-fold advantage in the TCP/BMP2 group compared with all of the other groups. Although previous biomechanical studies have evaluated screw fixation strength with pull-out testing (Upasani et al., 2009; Lapresle et al., 1995), a torsional screw extraction analysis was used in this study to specifically analyse the interaction between the implant and the vertebral body. We attribute the increased bond strength between the implanted screw and the pre-existing bone to increased bone formation at three sites: 1) around the screw, 2) within the screw, and 3) at the bridge between these sites of bone formation. The authors refer to the latter two sites as "bone-to-bone" fixation, which is unique to this surfacecoating method. This bone-to-bone fixation is a biological concept, whereas previous surface-coating techniques are considered non-biological, as bonding occurs between the host bone and non-biological material (screw or surfacecoating material). This assessment was confirmed by $\mu \mathrm{CT}$ analyses, which documented increased bone formation and also by histological examination that clearly showed new bone formation in and around the screw, demonstrating successful osseo-integration.

Although the previously reported titanium and hydroxyapatite plasma-spray-coated implants and cementing augmentation methods enhance fixation strength in the early stages, they sometimes fail to integrate with the pedicle screw and ultimately result in severe loosening. This loosening can be caused by inflammatory reactions, bone resorption, delamination between the hydroxyapatite coating and the implant (Lai et al., 2002), or secondary problems of bone defects due to non-biological implant materials. We designed the TCP/BMP2 loaded cannulated pedicle screw as a new concept for biologic fixation that allows for bone formation in the inner aspect of the pedicle screw. This provides increased fixation by forming a bond between the host bone surrounding the pedicle screw and the newly formed bone through the holes in the pedicle screws.

The most valuable result of this study is the demonstration of bone-to-bone fixation through the interface of the pedicle screw and host bone. This is a unique concept compared to previously reported methods, in terms of new implant to bone fixation. This bone-to-bone fixation can explain the markedly high radiological score and extraction torsional peak torque observed for the proposed method.

Osteolysis, increased risk of cancer, and inflammation are reported to be complications of rhBMP-2. No specific complication was found in this short-term study. One case of partial loosening was seen in the TCP/BMP2 loaded group, believed to be due to a lack of immobilisation of the miniature pig; however, the osteoclastic effect is also a possible cause of the partial loosening. The osteoclastic effect of rhBMP-2 is generally known to occur with a high dose of rhBMP-2 (AMPLIFY ${ }^{\circledR}$, Medtronic Sofamor Danek, USA). The possibility of loosening due to the osteoclastic effect is unlikely because a relatively low dose of $0.75 \mathrm{mg} /$ $\mathrm{mL}$ rhBMP-2 was used in this study. The overall high number of screw loosening cases in all groups may be attributed to uncontrolled postoperative immobilisation. There are still debates on the advantages and disadvantages of safety guidelines for rhBMP-2 usage. Preclinical data should be well documented before TCP/BMP2 loaded pedicle screws are applied to humans. Adequate indications should also be suggested.

Our studies also demonstrated the safety of this approach. There were no major operative or post-operative complications. There was one minor surgical wound infection in the treatment group that cannot be attributed to the treatment.

The major limitation of this study is authors did not utilise an osteoporotic model. The ultimate goal of this study was to show the advantage of TCP/BMP2 loaded pedicle screws in osteoporotic patients. However, the model we chose was not an osteoporotic model. The use of such a model would have produced more accurate results, as bone metabolism in osteoporotic bone is different from that of non-osteoporotic bone. The authors of this study focused on rhBMP-2-induced bone formation, while our next study will focus on an osteoporotic model.

Another limitation is statistical analysis. The randomised block design of inserting four different screws in one miniature pig is an accurate method for evaluating screw function. However, we inevitably use the method of inserting four screws in one miniature to exclude interactions among them due to newly formed bone. Therefore, this study has the limitation that the results of four screws in one miniature pig are considered as independent observations.

In summary, our study demonstrated improved integration of pedicle screws into pre-existing bone when the screw was cannulated and loaded with TCP and rhBMP-2. The authors do not necessarily recommend this type of approach for all clinical situations. It may not be required for routine surgery in young patients; however, it is likely to contribute to significantly improved outcomes in older patients with osteoporosis and in situations involving skeletal tumours.

\section{Conclusion}

Bone-to-bone biologic fixation through holes in TCP/ BMP2 loaded pedicle screws significantly promoted fixation strength and provides a potential future method for implant fixation that can be applied to osteoporotic or spinal tumour surgeries.

\section{Acknowledgements}

This study was supported by a grant from the CNUHBRI (Biomedical Research Institute of Chonbuk National University Hospital, CNUH-BRI-2012-02-005).

The authors thank Dr. Samuel S. Murray for his assistance with the English editing of the manuscript. 


\section{References}

Abul-Kasim K, Ohlin A. Evaluation of implant loosening following segmental pediclescrew fixation in adolescent idiopathic scoliosis: a 2 year follow-up with low-dose CT. Scoliosis 2014 Aug 24: 9: 13.

Bouxsein ML, Boyd SK, Christiansen BA, Guldberg RE, Jepsen KJ, Müller R (2010) Guidelines for Assessment of Bone Microstructure in Rodents Using Micro-Computed Tomography. J Bone Miner Res 25: 1468-1486.

Butler TE Jr, Asher MA, Jayaraman G, Nunley PD, Robinson RG (1994) The strength and stiffness of thoracic implant anchors in osteoporotic spines. Spine 19: 19561962.

DeCoster TA, Heetderks DB, Downey DJ, Ferries JS, Jones W (1990) Optimizing bone screw pullout force. J Orthop Trauma 4: 169-174.

Ferguson RL, Tencer AF, Woodard P, Allen BL Jr (1988) Biomechanical comparisons of spinal fracture models and the stabilizing effects of posterior instrumentations. Spine 13: 453-460.

Frankel BM, Jones T, Wang C (2007) Segmental polymethyl methacrylate augmented pedicle screw fixation in patients with bone softening caused by osteoporosis and metastatic tumor involvement: a clinical evaluation. Neurosurgery 61: 531-537; discussion 537-538.

Goel VK, Kim YE, Lim TH, Weinstein JN (1988) An analytical investigation of the mechanics of spinal instrumentation. Spine 13: 1003-1011.

Greenfield RT 3rd, Grant RE, Bryant D (1992) Pedicle screw fixation in the management of unstable thoracolumbar spine injuries. Orthop Rev 21: 701-706.

Halvorson TL, Kelley LA, Thomas KA, Whitecloud TS 3rd, Cook SD (1994) Effects of bone mineral density on pedicle screw fixation. Spine 19: 2415-2420.

Hitchon PW, Brenton MD, Black AG, From A, Harrod JS, Barry C, Serhan H, Torner JC (2003) In vitro biomechanical comparison of pedicle screws, sublaminar hooks, and sublaminar cables. J Neurosurg 99(Suppl 1): 104-109.

Johansson C, Albrektsson T (1987) Integration of screw implants in the rabbit: A 1-year follow-up of removal torque of titanium implants. Int J Oral Maxillofac Implants 2: 69-75.

Krag MH, Beynnon BD, Pope MH, Frymoyer JW, Haugh LD, Weaver DL (1986) An internal fixator for posterior application to short segments of the thoracic, lumbar, or lumbosacral spine. Design and testing.Clin Orthop Relat Res 203: 75-98.

Kwok AW, Finkelstein JA, Woodside T, Hearn TC, Hu RW (1996) Insertional torque and pull-out strengths of conical and cylindrical pedicle screws in cadaveric bone. Spine 21: 2429-2434.

Lai KA, Shen WJ, Chen CH, Yang CY, Hu WP, Chang GL (2002) Failure of hydroxyapatite-coated acetabular cups. Ten-year follow-up of 85 Landos Atoll arthroplasties. J Bone Joint Surg Br. 84: 641-646.

Lapresle P, Missenard G (1995) Hydroxylapatite-coated Diapason screws: first clinical report. J Spinal Disord 8 (Suppl 1):31-39.

McLain RF, Burkus JK, Benson DR (2001). Segmental instrumentation for thoracic and thoracolumbar fractures: prospective analysis of construct survival and five-year follow-up. Spine J 1: 310-323.

Ong JL, Carnes DL, Bessho K (2004) Evaluation of titanium plasma-sprayed and plasma-sprayed hydroxyapatite implants in vivo. Biomaterials 25: 46014606.

Sandén B, Olerud C, Petrén-Mallmin M, Johansson C, Larsson S (2004). The significance of radiolucent zones surrounding pediclescrews. Definition of screwloosening in spinal instrumentation. J Bone Joint Surg Br 86: 457-461.

Sandén B, Olerud C, Larsson S (2001) Hydroxyapatite coating enhances fixation of loaded pedicle screws: a mechanical in vivo study in sheep. Eur Spine J 10: 334-339.

Sell P, Collins M, Dove J (1988) Pedicle screws: axial pull-out strength in the lumbar spine. Spine 13: 1075-1076.

Upasani VV, Farnsworth CL, Tomlinson T, Chambers RC, Tsutsui S, Slivka MA, Mahar AT, Newton PO (2009). Pedicle Screw Surface Coatings Improve Fixation in Nonfusion Spinal Constructs. SPINE 34: 335-343.

Weinstein JN, Spratt KF, Spengler D, Brick C, Reid S (1988) Spinal pedicle fixation: reliability and validity of roentgenogram-based assessment and surgical factors on successful screw placement. Spine 13: 1012-1018.

Yuan HA, Garfin SR, Dickman CA, Mardjetko SM (1994) A historical cohort study of pedicle screw fixation in thoracic, lumbar, and sacral spinal fusions. Spine 19: 2279S-2296S.

Zdeblick TA (1993) A prospective, randomized study of lumbar fusion.Preliminary results. Spine 18: 983-991.

Zeiller SC, Lee J, Lim M, Vaccaro AR (2005) Posterior thoracic segmental pedicle screw instrumentation: evolving methods of safe and effective placement. Neurol India 53: 458-465.

Editor's Note: All questions/comments by the reviewers were answered by text changes. There is hence no Discussion with Reviewers section. 